Pure and Applied Mathematics Quarterly

Volume 2, Number 1

(Special Issue: In honor of

John H. Coates, Part 1 of 2)

$163-178,2006$

\title{
Towards Lang-Trotter for Elliptic Curves over Function Fields
}

\author{
Chris Hall and José Felipe Voloch
}

\section{INTRODUCTION}

Let $K$ be a global field of characteristic $p$ and let $\mathbb{F}_{q} \subset K$ denote the algebraic closure of $\mathbb{F}_{p}$ in $K$. We fix an elliptic curve $E / K$ with non-constant $j$-invariant and a torsion-free subgroup $\Sigma \subseteq E(K)$ of rank $r>0$. We write $V$ for the open set of places $v$ of $K$ such that the special fiber $E_{v}$ is an elliptic curve and, for $v$ in $V$, we let $\Sigma_{v} \subset E_{v}\left(k_{v}\right)$ be the image of $\Sigma$ under reduction modulo $v$, where $k_{v}$ is the residue field of $K$ at $v$. We fix a finite set of (rational) prime numbers $\mathcal{S}$ which is large enough to include the exceptional primes which we will define explicitly in section 2.4 and section 3), and we let $\mathcal{G}(\Sigma, \mathcal{S})$ denote the subset of $v \in V$ such that $\Sigma_{v}$ contains the prime-to- $\mathcal{S}$ part of $E_{v}\left(k_{v}\right)$. For every $n>0$, we write $V_{n}$ for the subset of $v \in V$ such that $\operatorname{deg}(v)=n$ and let $\mathcal{G}_{n}(\Sigma, \mathcal{S})=V_{n} \cap \mathcal{G}(\Sigma, \mathcal{S})$.

Theorem 1. Suppose $r \geq 6$. There exist constants a, $b$ satisfying $0<a<b<1$ and depending only on $r$ and $\mathcal{S}$ and for each $n \geq 1$, there exists $\delta_{n}(\Sigma, \mathcal{S})$, depending on $r, \mathcal{S}$ and the isomorphism class of $\operatorname{Gal}(K(E[\ell]) / K)$ for $\ell \notin \mathcal{S}$ such that

$$
a \leq \delta_{n}(\Sigma, \mathcal{S}) \leq b, \quad\left|\mathcal{G}_{n}(\Sigma, \mathcal{S})\right|=\delta_{n}(\Sigma, \mathcal{S})\left|V_{n}\right|+o\left(q^{n} / n\right)
$$

for all $n$.

We prove the theorem in section 4.3. The rest of the paper establishes preliminary results. We remark that for a fixed $K, r$ and $\mathcal{S}$, there are only finitely many possibilities for the entire set $\left\{\delta_{n}(\Sigma, \mathcal{S}): n>0\right\}$, as will follow from our

Received June 1, 2005.

The first author was an NSF VIGRE postdoc and the second author was supported by NSA grant MDA904-03-1-0117. 
results. However, the limit $\delta_{n}(\Sigma, \mathcal{S})$ as $n \rightarrow \infty$ does not exist in general. Rather one must consider an increasing sequence $n_{1}, n_{2}, \ldots$ such that $n_{i}$ divides $n_{i+1}$ in order to obtain a limit. Different sequences will lead to different limits. This is in contrast with the number field analogue in which the analogous $\delta_{n}$ converge and therefore can be taken independent of $n$.

The number field analogue of the above theorem was conjectured by Lang and Trotter (for $r=1$ ) and proved by Gupta and Murty ([GM]) for $r \geq 18$ under GRH. Our proof of theorem 1 follows the function-field analogue of the strategy in $[\mathrm{GM}]$. A literal translation of their argument, together with the improved error bounds of [MS], allows one to prove an analogue of their theorem for $r \geq 10$. The proof proceeds in several stages, and in one of these stages we replace the use of $K(E[\ell])$ with a smaller subextension of $K$, which we define in section 2.2. This allows us to extend the argument to $r \geq 6$. It is conceivable that these extensions might be used in the number field case to lower the bound on $r$. We hope to return to this question in a later paper. The case of constant $j$-invariant and $r=1$ was treated by the second author in [V]. One can always construct examples where the hypotheses of the theorem apply by passing to an extension of $K$, if necessary. If one insists on examples over the rational function field $\mathbb{F}_{q}(t)$, these have been constructed by Ulmer ([U]).

\section{2. $\ell$-ADiC Galois TheORY}

Throughout this section $K$ is an arbitrary field and $\ell$ is a fixed rational prime which is invertible in $K$. We fix an elliptic $E / K$ and write $L=K(E[\ell])$. There is an embedding $\Gamma=\operatorname{Gal}(L / K) \rightarrow \mathrm{GL}_{2}(\mathbb{Z} / \ell)$, which is well-defined, up to conjugation, and is given by identifying $\operatorname{Aut}(E[\ell])$ with $\mathrm{GL}_{2}(\mathbb{Z} / \ell)$. Moreover, for a fixed primitive $\ell$ th root of unity $\zeta$, the quotient $\operatorname{det}(\Gamma)$ is the Galois group of the cyclotomic extension $K(\zeta) / K$. We call the kernel of $\Gamma \rightarrow \operatorname{det}(\Gamma)$ the subgroup of geometric elements, or simply the geometric Galois group of $L / K$. In all but the last section we assume that it is $\mathrm{SL}_{2}(\mathbb{Z} / \ell)$. In the last section we consider the case when the geometric Galois group is a proper subgroup of $\mathrm{SL}_{2}(\mathbb{Z} / \ell)$.

2.1. Kummer Theory. In this section we additionally assume $\ell>2$. Recall $L=K(E[\ell])$ and $\Gamma=\operatorname{Gal}(L / K)$ contains $\mathrm{SL}_{2}(\mathbb{Z} / \ell)$.

Lemma 1. The natural map $E(K) / \ell(E(K)) \rightarrow E(L) / \ell(E(L))$ is injective.

Proof. For every finite extension $F / K$ there is a natural embedding of $E(F) / \ell(E(F))$ into the Galois cohomology group $H^{1}(F, E[\ell])$, hence it suffices to show that 
the restriction map $H^{1}(K, E[\ell]) \rightarrow H^{1}(L, E[\ell])$ is injective. By the inflationrestriction sequence, the kernel of the restriction map is $H^{1}(\Gamma, E[\ell])$. Let $Z$ be the center of $\Gamma$; it is a normal subgroup of order prime to $\ell$ and $E[\ell]^{Z}=0$. It follows that $H^{1}(\Gamma, E[\ell])=0$, proving the lemma.

For any $P \in E(K)$, we write $P / \ell$ for the $\ell^{2}$ points $Q$ such that $[\ell] Q=P$. The extension $K(P / \ell) / K$ is Galois and contains $L$, and we write $H=\operatorname{Gal}(L(P / \ell) / L)$, $G=\operatorname{Gal}(K(P / \ell) / K)$. There is a short exact sequence of groups

$$
1 \longrightarrow H \longrightarrow G \longrightarrow \Gamma \longrightarrow 1 \text {. }
$$

We regard $E[\ell]$ as a $\Gamma$-module and write $E[\ell] \rtimes \Gamma$ for the semi-direct product. There is an embedding $G \rightarrow E[\ell] \rtimes \Gamma$, which is unique up to conjugation in $E[\ell] \rtimes \Gamma$, and $H=G \cap E[\ell]$.

Lemma 2. For every $P \in E(K)-\ell(E(K)), G=\operatorname{Gal}(K(P / \ell) / K)$ is isomorphic to $E[\ell] \rtimes \Gamma$.

Proof. A priori $G$ is a subgroup of $E[\ell] \rtimes \Gamma$ which maps surjectively to $\Gamma$. No line of $E[\ell]$ is stabilized by $\Gamma$, so $H=G \cap E[\ell]$ is either trivial or all of $E[\ell]$. Lemma 1 implies that $P$ is not in $\ell(E(L))$, hence $H=E[\ell]$.

The lines $\mathcal{K} \subset E[\ell]$ correspond bijectively to cyclic $\ell$-isogenies $\phi: E_{\phi} \rightarrow E$, where $E_{\phi}$ is some elliptic curve. Given $\mathcal{K}, \phi$ is the dual of the canonical map $E \rightarrow E / \mathcal{K}$ and, given $\phi: E_{\phi} \rightarrow E, \mathcal{K}$ is the kernel of the dual isogeny $E \rightarrow E_{\phi}$.

Lemma 3. Let $P, Q \in E(K)$. The following are equivalent:

(1) $\langle P\rangle \equiv\langle Q\rangle \bmod \ell(E(K))$;

(2) $\langle P\rangle \equiv\langle Q\rangle \bmod \ell(E(L))$;

(3) $\langle P\rangle \equiv\langle Q\rangle \bmod \phi\left(E_{\phi}(L)\right)$ for every cyclic $\ell$-isogeny $\phi: E_{\phi} \rightarrow E$;

(4) $\langle P\rangle \equiv\langle Q\rangle \bmod \phi\left(E_{\phi}(L)\right)$ for some cyclic $\ell$-isogeny $\phi: E_{\phi} \rightarrow E$.

Proof. The first two statements are equivalent by lemma 1 . The second statement implies the third. Given an $\ell$-isogeny $\phi: E_{\phi} \rightarrow E$ we have an exact sequence

$$
0 \rightarrow E[\hat{\phi}] \rightarrow E[\ell] \rightarrow E_{\phi}[\phi] \rightarrow 0
$$

and the cohomology sequence gives the short exact sequence

$$
0 \rightarrow H^{1}(L, E[\hat{\phi}]) \rightarrow H^{1}(L, E[\ell]) \rightarrow H^{1}\left(L, E_{\phi}[\phi]\right) .
$$

For isogenies $\phi_{1} \neq \phi_{2}$, the intersection $H^{1}\left(L, E\left[\hat{\phi}_{1}\right]\right) \cap H^{1}\left(L, E\left[\hat{\phi}_{2}\right]\right)$ is trivial, as $H^{1}(L, E[\ell])$ is a direct sum of these two subgroups, therefore the composite map

$$
E(L) / \ell(E(L)) \rightarrow H^{1}(L, E[\ell]) \rightarrow H^{1}\left(L, E_{\phi_{1}}\left[\phi_{1}\right]\right) \oplus H^{1}\left(L, E_{\phi_{2}}\left[\phi_{2}\right]\right)
$$


is injective. It is the direct sum of the boundary maps corresponding to the cohomology sequence of

$$
0 \rightarrow E_{\phi_{i}}\left[\phi_{i}\right] \rightarrow E_{\phi_{i}} \stackrel{\phi_{i}}{\rightarrow} E \rightarrow 0
$$

These maps induce embeddings $E(L) / \phi_{i} E_{\phi_{i}}(L) \rightarrow H^{1}\left(L, E_{\phi_{i}}\left[\phi_{i}\right]\right)$. If we assume the third statement of the lemma holds, then $\langle P\rangle \equiv\langle Q\rangle$ in all the terms of (1), hence the second statement holds. The last two statements of the lemma are equivalent because $\Gamma$ acts transitively on the isogenies and fixes $P$ and $Q$.

One useful aspect of this lemma is that, in some circumstances, it allows us to replace $L$ with the field of definition $K(\phi)$, for a fixed $\phi$ of our choosing (cf. section 2.2). We can also apply the lemma to the Galois theory of ' $\ell$-descent' of $E / K$.

Theorem 2. Let $P, Q \in E(K)$. The following are equivalent:

(1) $K(P / \ell)=K(Q / \ell)$;

(2) $\langle P\rangle \equiv\langle Q\rangle \bmod \ell(E(K))$.

Otherwise $K(P / \ell) \cap K(Q / \ell)=L$.

Proof. The statement follows easily from lemma 2 when $P$ or $Q$ lies in $\ell(E(K))$, so we assume that $P, Q \in E(K)-\ell(E(K))$. We also assume there exists $F \subset$ $K(P / \ell) \cap K(Q / \ell)$ which is a non-trivial extension of $L$ of degree $\ell . F$ is not Galois over $K$ because $E[\ell] \rtimes \Gamma$ has no normal subgroups of order $\ell$, hence $K(P / \ell)=K(Q / \ell) . \operatorname{Gal}(L(P / \ell) / L)=\operatorname{Gal}(L(Q / \ell) / L)$ is isomorphic to $E[\ell]$, so the Galois group $\operatorname{Gal}(F / L)$ is isomorphic to $E_{\phi}[\phi]$ for some cyclic $\ell$-isogeny $\phi: E_{\phi} \rightarrow E$. The kernel of the restriction map $H^{1}\left(L, E_{\phi}[\phi]\right) \rightarrow H^{1}\left(F, E_{\phi}[\phi]\right)$ is isomorphic to $\mathbb{Z} / \ell$, and it is generated by the image of $P, Q$ under the boundary map $E(L) \rightarrow H^{1}\left(L, E_{\phi}[\phi]\right)$. Therefore $\langle P\rangle \equiv\langle Q\rangle \bmod \phi\left(E_{\phi}(L)\right)$, hence $\langle P\rangle \equiv\langle Q\rangle \bmod \ell(E(K))$ by lemma 3 , so we have the implication $(1) \Rightarrow(2)$. The converse implication is clear.

For any $d \geq 0, \Gamma$ acts diagonally on $E[\ell]^{d}$, and we write $E[\ell]^{d} \rtimes \Gamma$ for the semi-direct product.

Corollary 1. If the image of $P_{1}, \ldots, P_{r} \in E(K)$ in $E(K) / \ell(E(K))$ generates a $d$-dimensional subspace, then $\operatorname{Gal}\left(K\left(P_{1} / \ell, \ldots, P_{r} / \ell\right) / K\right) \simeq E[\ell]^{d} \rtimes \Gamma$.

In general we will apply this for fixed $P_{1}, \ldots, P_{r}$ and varying $\ell$ in the proof of theorem 1 . One can prove analogous results for any cyclic $\ell$-isogeny. 
Theorem 3. Let $\phi: E_{\phi} \rightarrow E$ be a cyclic $\ell$-isogeny. For $P \in E(K)$ let $P / \phi$ denote the set of $\ell$ points $Q$ such that $\phi(Q)=P$. If the images of $P_{1}, \ldots, P_{r} \in E(K)$ in $E(K(\phi)) / \phi\left(E_{\phi}(K(\phi))\right)$ generate a d-dimensional subspace, then

$$
\operatorname{Gal}\left(K\left(\phi, P_{1} / \phi, \ldots, P_{r} / \phi\right) / K(\phi)\right) \simeq E_{\phi}[\phi]^{d} \rtimes \operatorname{det}(\Gamma) .
$$

We will be most interested in applying this with $r=1$ in the proof of lemma 7 .

2.2. Cyclotomic Twist. In this section we fix a cyclic $\ell$-isogeny $\phi: E_{\phi} \rightarrow E$. We let $\mathcal{K}=\operatorname{Ker}(\hat{\phi})=\phi\left(E_{\phi}[\ell]\right)$ and write $B \subset \Gamma$ for the unique Borel subgroup stabilizing $\mathcal{K}$. We may assume, without loss of generality, that $B$ is the subgroup of upper-triangular matrices. Then the $\ell$-Sylow subgroup $U \subset B$ is the subgroup of upper-unipotent matrices. We choose a second Borel subgroup $\hat{B} \neq B$. The intersection $C=B \cap \hat{B}$ is a (split) Cartan subgroup, and $B$ is then canonically isomorphic to the semi-direct product $U \rtimes C$. Up to conjugation by an element of $U$, we may assume, without loss of generality, that $\hat{B}$ is the subgroup of lowertriangular matrices, so $C \subset \Gamma$ is the subgroup of diagonal matrices. We write $\hat{\mathcal{K}} \subset E[l]$ for the unique line stabilized by $\hat{B}$, and we note that $\mathcal{K} \neq \hat{\mathcal{K}}$, hence $\hat{\phi}: \hat{\mathcal{K}} \rightarrow E_{\phi}[\phi]$ is an isomorphism.

We define $\hat{T}, T \subset C$, respectively, to be the subgroups which act trivially on $\mathcal{K}, \hat{\mathcal{K}}$, respectively. We note that the semi-direct products $U \rtimes T, U \rtimes \hat{T} \subset B$ are each stable under conjugation by $U$, hence are independent of our choice of $\hat{B}$. We define the geometric subgroup $G=\hat{G} \subset C$ to be the kernel of $C \rightarrow \operatorname{det}(C)$. The fixed field of $G$ is the cyclotomic extension $K(\phi, \zeta) / K(\phi)$, where $\zeta$ is a primitive $\ell$ th root of unity. The multiplication maps $T \times G \rightarrow C$ and $\hat{T} \times \hat{G} \rightarrow C$ are isomorphisms, so there are canonical isomorphisms $G \rightarrow \operatorname{Gal}\left(K\left(\phi, E_{\phi}[\phi]\right) / K(\phi)\right)$ and $\hat{G} \rightarrow \operatorname{Gal}(K(\hat{\phi}, E[\hat{\phi}]) / K(\hat{\phi}))$. In summary, we have the lattice of Galois extensions shown in figure 1.

The extension $N / K$ is an instance of the 'balanced- $\Gamma_{1}(\ell)$-moduli problem' of (7.4.3) of $[\mathrm{KM}]$. That is, it classifies pairs of embeddings $\mathbb{Z} / \ell \rightarrow E[\ell], \mathbb{Z} / \ell \rightarrow$ $E_{\phi}[\ell]$ of the trivial Galois module $\mathbb{Z} / \ell$. Similarly, $\hat{F} / K$ is an instance of the ' $\Gamma_{1}(\ell)$-moduli problem' of loc. cit., which classifies embeddings of $\mathbb{Z} / \ell$ into $E[\ell]$. The inclusion of fields $\hat{F} \rightarrow N$ corresponds to 'remembering' the embedding $\mathbb{Z} / \ell \rightarrow E_{\phi}[\ell]$. One can also consider embeddings $\mu_{\ell} \rightarrow E[\ell]$, where $\mu_{\ell}$ is the Galois module of $\ell$ th roots of unity. By Cartier duality these correspond to quotients $E[\ell] \rightarrow \mathbb{Z} / \ell$, hence embeddings $\mathbb{Z} / \ell \rightarrow E_{\phi}[\ell]$. The extension $F / K$ corresponds to an instance of this other 'moduli problem,' and $F \rightarrow N$ corresponds to 'remembering' the embedding $\mathbb{Z} / l \rightarrow E[\ell]$. As 'moduli problems,' these last two are isomorphic if and only if $\mu_{\ell}$ and $\mathbb{Z} / \ell$ are isomorphic Galois modules; that is, 
Figure 1

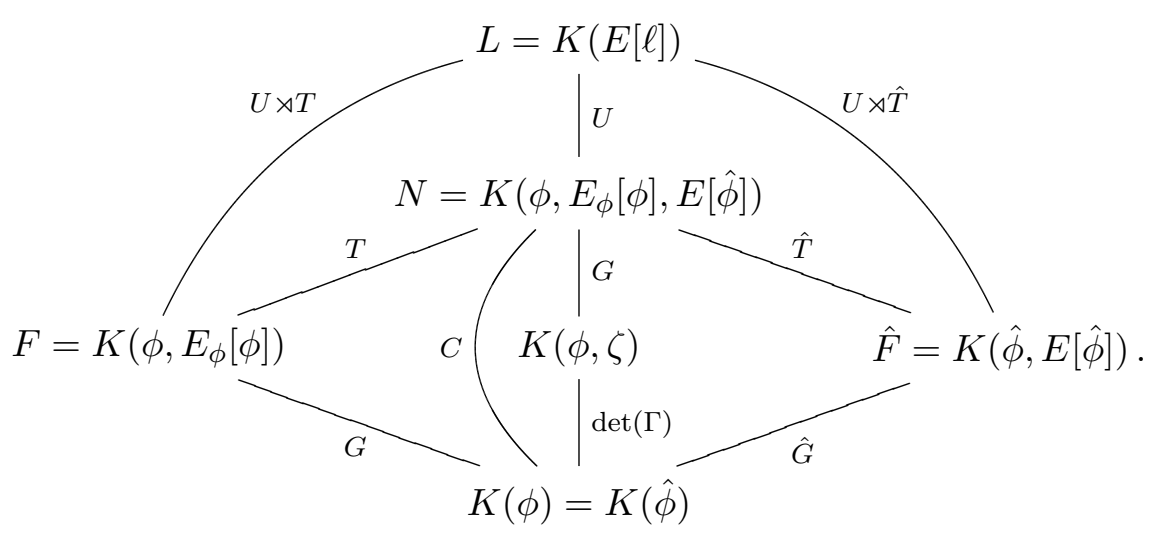

Figure 2

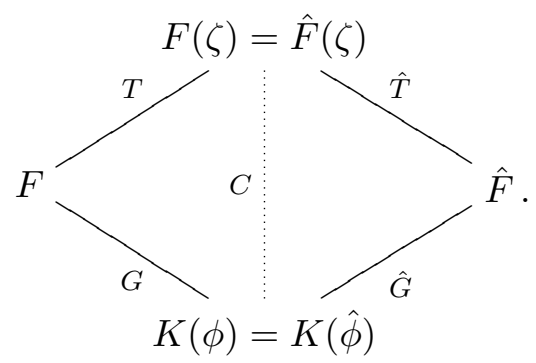

they classify the same objects if and only if $K(\zeta)=K$. Otherwise $\hat{F}$ is an easily described 'cyclotomic twist' of $F$ (and vice versa).

We observe that $F / K, \hat{F} / K$ are geometric extensions, because $\operatorname{det}(U \rtimes T)=$ $\operatorname{det}(U \rtimes \hat{T})=\operatorname{det}(\Gamma)$. On the other hand, the extension $N=F(\zeta)=\hat{F}(\zeta)$ of $F, \hat{F}$, respectively, is 'purely arithmetic'. Hence we can extract the 'Cartesian square' of Galois field extensions in figure 2 from the lattice in figure 1 . There is a canonical isomorphism between Galois groups for either pair of parallel edges, and there is one, $T \rightarrow \hat{T}$, induced by the isomorphisms $T \rightarrow \operatorname{det}(C)$ and $\hat{T} \rightarrow$ $\operatorname{det}(C)$. Composing the canonical maps $T \rightarrow \hat{T}$ and $\hat{T} \rightarrow G$ gives a 1-cocycle $\sigma \in H^{1}(T, G) \subset H^{1}(F, G)$. One can easily verify that $\hat{T} \subset C$ is the graph of $\sigma$ in $T \times G=C$, hence $\hat{F}$ is the 'cyclotomic twist' corresponding to $\sigma$. 
Figure 3

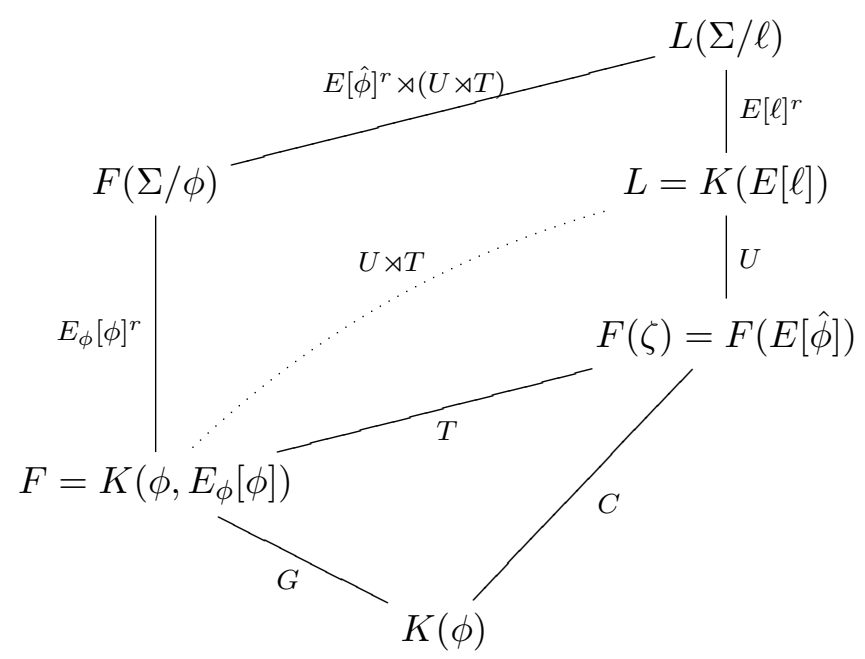

2.3. Lang-Trotter Conjugacy Classes. We continue to use the notation of the previous two sections. We fix a free subgroup $\Sigma=\left\langle P_{1}, \ldots, P_{r}\right\rangle \subset E(K)$ of rank $r$. We assume its image is an $r$-dimensional subspace of $E(K) / \ell(E(K))$, so that $K(E[\ell], \Sigma / \ell)$ is Galois over $K$ with group $E[\ell]^{r} \rtimes \Gamma$ (by corollary 1). Then for every $\phi$, we have the lattice of Galois extensions in figure 3.

We define the Lang-Trotter elements of $\Gamma$ associated to $\phi$ by

$$
\mathcal{C}(\phi)=\{\tau \in U \rtimes T: \tau=1 \text { or } \tau \notin U\} .
$$

That is, $\mathcal{C}(\phi) \subset U \rtimes T$ is the subset of semisimple elements. We define $\mathcal{C}(\phi, \Sigma)$ to be the inverse image of $\mathcal{C}(\phi)$ under the natural map $E[\hat{\phi}]^{r} \rtimes(U \rtimes T) \rightarrow U \rtimes T$. It is important to note that every element of $\mathcal{C}(\phi, \Sigma)$ acts trivially on $F(\Sigma / \phi)$ (cf. beginning of section 4.3). The subsets of Lang-Trotter conjugacy classes are the unions $\mathcal{C}=\cup_{\phi} \mathfrak{C}(\phi), \mathcal{C}(\Sigma)=\cup_{\phi} \mathfrak{C}(\phi, \Sigma)$ over all $\phi$. One can easily show that for every $\delta \in \operatorname{det}(\Gamma)$,

$$
|\{c \in \mathcal{C}(\Sigma): \operatorname{det}(c)=\delta\}|=\left\{\begin{array}{ll}
\ell^{r+1}(\ell+1) & \text { if } \delta \neq 1 \\
\ell\left(\ell^{r}+\ell^{r-1}-1\right) & \text { if } \delta=1
\end{array} .\right.
$$

When $\delta=1$ we remark that $\mathcal{C}(\phi)=\mathcal{C}\left(\phi^{\prime}\right)=\{1\}$ and $\mathcal{C}(\phi, \Sigma) \cap \mathcal{C}\left(\phi^{\prime}, \Sigma\right)=\{(0,1)\}$ for $\phi \neq \phi^{\prime}$. We note that for every $\delta$ there is at least one element of $E[\ell]^{r} \rtimes \Gamma$ which does not lie in $\mathcal{C}(\Sigma)$ and whose image in $\Gamma$ has determinant $\delta$. 
For the rest of this section we fix a place $v$ of $K$ which is unramified in $L$ and let $n=\operatorname{deg}(v)$. The prime decomposition of $v$ in the extension $L / K$ is relatively easy to describe, mainly because the latter is Galois. Let $w$ be any prime of $L$ lying over $v$ and let $D(w) \subset \Gamma$ denote the decomposition group. There are $[\Gamma: D(w)]$ primes $w$ over $v$, all of degree $f=|D(w)|$. $\Gamma$ acts transitively on the $w$ over $v$, and the induced action on $\{D(w)\}$ is conjugation. On the other hand, the prime decomposition of $v$ in $K(\phi)$ is more difficult to describe because $K(\phi) / K$ is not a Galois extension. We will refrain from describing the general case, and instead will assume that the Frobenius conjugacy class $\operatorname{Fr}_{v} \subset \Gamma$ is contained in $\mathcal{e} \subset \Gamma$.

For any prime $w$ over $v$, we write $\bar{w}$ for the prime in $K(\phi)$ under $w$. The decomposition group $D(\bar{w}) \subset B$ is the intersection $D(w) \cap B$, hence $\bar{w}$ has degree $[D(w): D(\bar{w})]$ over $v$ If $f=1$, then $D(w)$ is trivial, hence $v$ decomposes as a product of $\ell+1$ primes of degree $n$ in $K(\phi)$. On the other hand, if $f>1$, then $D(w)$ is contained in a unique split Cartan subgroup $C(w) \subset \Gamma ; C(w)$ is the centralizer of $D(w)$ in $\Gamma$. Therefore $D(w)=D(\bar{w})$ if and only if $C=C(w) \subset B$, otherwise $D(\bar{w})=\{1\}$. Finally, $C$ has index two in its normalizer $N(C)$ and the intersection $B \cap N(C)$ is contained in $C$. Therefore the $\bar{w}$ such that $D(\bar{w}) \subset B$ lie in two $B$-orbits, one for each element of the intersection $\operatorname{Fr}_{v} \cap C$, hence there are two $\bar{w}$ of degree $n$ and the remaining $\bar{w}$ are of degree $n f$.

2.4. Geometrically-Degenerate Case. In this section we relax the condition that $\Delta=\operatorname{Gal}(L / K)$ contain the subgroup $\mathrm{SL}_{2}(\mathbb{Z} / \ell)$. More precisely, we assume the geometric subgroup, $\Delta \cap \mathrm{SL}_{2}(\mathbb{Z} / \ell)$, is a proper subgroup of $\mathrm{SL}_{2}(\mathbb{Z} / \ell)$. We fix a free subgroup $\Sigma=\left\langle P_{1}, \ldots, P_{r}\right\rangle \subset E(K)$ and let $H=\operatorname{Gal}(K(\Sigma / \ell) / K)$. In order to keep with notation of the previous sections, we write $\Gamma \subset \mathrm{GL}_{2}(\mathbb{Z} / \ell)$ for the inverse image of $\operatorname{det}(\Delta) \subset(\mathbb{Z} / \ell)^{\times}$. Then $\Delta$ is a proper subgroup of $\Gamma$ and $H$ is a proper subgroup of $G=E[\ell]^{r} \rtimes \Gamma$.

For every Borel subgroup $B \subset \Gamma$, we write $U \rtimes T \subset B$ for the 'semi-Borel subgroup' of section 2.3, and $\mathcal{K} \subset E[\ell]$ for the line stabilized by $B$. The LangTrotter elements associated to $B$ are the semisimple elements $\mathcal{C}(B) \subset U \rtimes T$, and the set of elements associated to $\Gamma$ is the union $\mathcal{C}=\cup_{B} \mathcal{C}(B)$ over all $B$. We recall that $U \rtimes T$ stabilizes $\mathcal{K}$, so the semi-direct product $\mathcal{K}^{r} \rtimes(U \rtimes T)$ exists. Then we define $\mathcal{C}(B, \Sigma)$ to be the inverse image of $\mathcal{C}(B)$ under the natural map $\mathcal{K}^{r} \rtimes(U \rtimes T) \rightarrow U \rtimes T$ and $\mathcal{C}(\Sigma) \subset G$ to be the union $\cup_{B} \mathcal{C}(B, \Sigma)$. We define the Lang-Trotter conjugacy classes $\mathrm{C}(H) \subset H$ as the intersection $H \cap \mathcal{C}(\Sigma)$.

Because $\Delta$ is a proper subgroup of $\Gamma$ such that $\operatorname{det}(\Delta)=\operatorname{det}(\Gamma)$, one can easily show that $|\mathcal{C}(H)|$ is maximized when $H=E[\ell]^{r} \rtimes C$ for some split Cartan 
subgroup $C \subset \Gamma$. Therefore, in general,

$$
\begin{aligned}
|\{c \in \mathcal{C}(H): \operatorname{det}(c)=\delta\}| & \leq\left|\left\{c \in \mathcal{C}\left(E[\ell]^{r} \rtimes C\right): \operatorname{det}(c)=1\right\}\right| \\
& \leq \ell\left(\ell^{r}+\ell^{r-1}-1\right),
\end{aligned}
$$

for any $\delta \in \operatorname{det}(\Gamma)$. A priori, every element of $H$ or simply every element of a fixed determinant $\delta \in \operatorname{det}(\Gamma)$ may be a Lang-Trotter element, in which case we call $\ell$ exceptional and therefore assume it is contained in the set $\mathcal{S}$ (cf. introduction) This applies to $\ell \neq p$ and the case $\ell=p$ is discussed in the next section. For any fixed $E / K$ the function-field analogue of Serre's theorem implies that there are only finitely many exceptional $\ell$. In fact, by theorem 1 of $[\mathrm{CH}]$ there is constant $\ell_{0}$, depending only on the genus of $K$, such that $\ell \leq \ell_{0}$ if $\ell$ is exceptional and $\ell \neq p$.

\section{3. $p$-ADiC Galois TheORY}

In this section we fix a global field $K$ of char $p$ and an elliptic curve $E / K$ with non-constant $j$-invariant. Then $E[p]$ is isomorphic to $\mathbb{Z} / p$ over an algebraic closure of $K$. There is a canonical cyclic $p$-isogeny $V: E_{\phi} \rightarrow E$ over $K$, the so-called Verschiebung; the dual isogeny $\hat{V}: E \rightarrow E_{\phi}$ is the $(p-)$ Frobenius While $K(E[p]) / K$ is inseparable in general, the extension $L=K\left(E_{\phi}[\phi]\right) / K$ is Galois and geometric, and there is an embedding of $\Delta=\operatorname{Gal}(L / K)$ into $(\mathbb{Z} / p)^{\times}$.

Lemma 4. The canonical map $H^{1}\left(K, E_{\phi}[V]\right) \rightarrow H^{1}\left(L, E_{\phi}[V]\right)^{\Delta}$ is an isomorphism.

Proof. The order of $\Delta$ is prime to $p$, so we consider the Hochschild-Serre sequence

$$
0 \longrightarrow H^{1}\left(\Delta, E_{\phi}[V]\right) \longrightarrow H^{1}\left(K, E_{\phi}[V]\right) \longrightarrow H^{1}\left(L, E_{\phi}[V]\right)^{\Delta} \longrightarrow H^{2}\left(\Delta, E_{\phi}[V]\right) \text {. }
$$

The first and last terms vanish, so the sequence degenerates to the desired isomorphism.

From the lemma we infer that $P \in V\left(E_{\phi}(K)\right)$ if and only if $P \in V\left(E_{\phi}(L)\right)$, which is what we need to prove the following theorem.

Theorem 4. Let $P_{1}, \ldots, P_{r} \in E(K)$. Suppose the image of $\left\langle P_{1}, \ldots, P_{r}\right\rangle$ in $E(K) / V\left(E_{\phi}(K)\right)$ is an $r$-dimensional subspace. Then

$$
\operatorname{Gal}\left(K\left(P_{1} / V, \ldots, P_{r} / V\right) / K\right) \simeq E_{\phi}[V]^{r} \rtimes \Delta .
$$

Finally, we define the Lang-Trotter conjugacy classes in $E_{\phi}[V]^{r} \rtimes \Delta$ to be the subgroup $E_{\phi}[V]^{r}$. Therefore, if $\Delta=1$, then we say that $p$ is exceptional and 
therefore must be added to our set $\mathcal{S}$ (cf. introduction). Contrary to the $\ell$-adic case, where there is a natural determinant det $: \Delta \rightarrow(\mathbb{Z} / \ell)^{\times}$, there are two natural maps det : $\Delta \rightarrow(\mathbb{Z} / p)^{\times}$that we must consider: the identity map and the trivial map. In fact, the latter is what we want if we insist that $\operatorname{det}(\Gamma)$ should be the Galois group of the scalar part of $L / K$, hence is trivial because $L / K$ is geometric.

\section{Chebotarev Argument}

4.1. Notation. We write $f(x)=O(g(x))$, as usual, to indicate that there is a constant $c>0$ such that $f(x)<c \cdot g(x)$, for all $x$. Moreover, we assume that $c$ depends at most on the genus of $K, \operatorname{deg}(S)$, and the 'regulator' $R=\operatorname{det}(\Sigma)$. We remark that the only place $R$ appears is in the proof of lemma 9 . We write $f(x)=o(g(x))$ to indicate that $f(x) / g(x)$ tends to 0 as $x$ tends to $\infty$.

4.2. Weil and Murty-Scherk Bounds. There is a finite set of places $S$ of $K$ such that $K(\Sigma / \ell) / K$, a fortiori $K(E[\ell]) / K$, is unramified away from $S$ for every $\ell \neq p$. On the other hand, if $V: E^{(p)} \rightarrow E$ is the Verschiebung, then $K(\Sigma / V) / K$ is unramified away from a divisor of degree $O(p \operatorname{deg}(S))$. In particular, every extension we encounter in this section will be unramified away from a divisor of uniformly bounded degree $d$, even tamely ramified, hence the following lemma will be useful.

Lemma 5. If $F / K$ is a tame extension which is unramified away from a divisor of degree at most d, then the genus of $F$ is $O([F: K])$.

Proof. This follows immediately from the Riemann-Hurwitz formula for the extension $F / K$ :

$$
2 \cdot \operatorname{genus}(F)-2=[F: K](2 \cdot \operatorname{genus}(K)-2)+(\text { ramification part }) .
$$

The ramification term part is at most $d([F: K]-1)$.

Let $V$ denote the open complement of $S$ and $V_{n} \subset V$ the subset of $v$ such that $\operatorname{deg}(v)=n$. One effective Chebotarev theorem we need is a simple form of the Weil bound.

Theorem 5 (Weil). Suppose $F / K$ is a tame, finite Galois and geometric extension, which is unramified away from $S$, and $L / K$ is a subextension. Let $W_{n}$ denote the subset of places $w$ of $L$ of degree $n$. Then for any $n \geq 1$,

$$
\mid\left\{w \in W_{n}: w \text { splits completely in } F / L\right\}\left|=\frac{1}{[F: L]}\right| W_{n} \mid+O\left([L: K] \frac{q^{n / 2}}{n}\right) .
$$


We note that the geometric assumption is crucial, for otherwise none of the points in $W_{n}$ split completely for general $n$. The factor $[L: K]$ in the error term accounts for the genus of $L$.

Suppose $L / K$ is a geometric subextension of the finite Galois extension $F / K$. Let $G=\operatorname{Gal}(F / L)$. For every place $w$ of $L$, unramified in $F$, there is a welldefined conjugacy class $\operatorname{Fr}_{w} \subset G$, the so-called Frobenius class. Let $\mathbb{F}_{q^{m}} \subset F$ be the algebraic closure of $\mathbb{F}_{q} \subset K$. By assumption $\mathbb{F}_{q^{m}} \cap L=\mathbb{F}_{q}$, and there is a short exact sequence

$$
1 \longrightarrow \operatorname{Gal}\left(F / \mathbb{F}_{q^{m}} L\right) \longrightarrow G \longrightarrow \operatorname{Gal}\left(\mathbb{F}_{q^{m}} / \mathbb{F}_{q}\right) \longrightarrow 1 .
$$

We write $G\left(q^{n}\right) \subset G$ for the subset of elements whose image in $\operatorname{Gal}\left(\mathbb{F}_{q^{m}} / \mathbb{F}_{q}\right)$ is the $n$th power of the Frobenius element. It is closed under conjugation by any element of $G$. Similarly, for any union of conjugacy classes $\mathcal{C} \subset G$, we write $\mathcal{C}\left(q^{n}\right)$ for the intersection $\mathcal{C} \cap G\left(q^{n}\right)$.

Theorem 6 (Murty-Scherk). Suppose $F / K$ is a tame, finite Galois extension which is unramified away from $S$, and $L / K$ is a geometric subextension. Let $G=\operatorname{Gal}(F / L)$ and let $W_{n}$ denote the subset of places $w$ of $L$ of degree $n$. Suppose $\mathcal{C} \subset G$ is a union of conjugacy classes. Then for $n \geq 1$,

$$
\left|\left\{w \in W_{n}: \operatorname{Fr}_{w} \subset \mathcal{C}\left(q^{n}\right)\right\}\right|=\frac{\left|\mathcal{C}\left(q^{n}\right)\right|}{\left|G\left(q^{n}\right)\right|}\left|W_{n}\right|+O\left([L: K] \cdot\left|\mathcal{C}\left(q^{n}\right)\right|^{1 / 2} q^{n / 2} / n\right) .
$$

Except for the tameness condition, this is essentially theorem 2 in [MS]. As before, the factor $[L: K]$ in the error term accounts for the genus of $L$.

4.3. Proof of Theorem 1. We write $V_{n}$ for the subset of places $v$ of $K$ such that $\operatorname{deg}(v)=n$. Let $\mathcal{L}$ denote the rational primes excluding $\mathcal{S}$ and let $\mathcal{F}$ denote the positive integers which are a square-free product of primes in $\mathcal{L}$. We write $\mathcal{G}_{n} \subset V_{n}$ for the subset of places $v$ which are good with respect to $\mathcal{L}$, that is, for which $\Sigma_{v}$ contains the prime-to- $\mathcal{S}$ part of $E_{v}$. Similarly, for any $f \in \mathcal{F}$, we write $\mathcal{B}_{n}(f) \subset V_{n}$ for the subset of $v$ which are bad with respect to every $\ell$ dividing $f$.

For every $\ell \in \mathcal{L}, \ell \neq p$, we write $K_{\ell}$ for the extension $K(\Sigma / \ell) / K$. Similarly, for $\ell=p$, we write $K_{p}$ for the extension $K(\Sigma / V) / K$, where $V: E^{(p)} \rightarrow E$ is the Verschiebung. In either case we let $G_{\ell}$ denote $\operatorname{Gal}\left(K_{\ell} / K\right)$ and $\mathcal{C}_{\ell} \subset G_{\ell}$ the subset of Lang-Trotter conjugacy classes. For every $v \in V_{n}$, we write $\operatorname{Fr}_{v} \subset G_{\ell}$ for the Frobenius conjugacy class. If we write $\mathcal{C}_{\ell}=\cup_{\phi} \mathcal{C}(\phi, \Sigma)$ (cf. section 2.3), then it follows from the definition of $\mathcal{C}(\phi, \Sigma)$ that $\operatorname{Fr}_{v} \cap \mathcal{C}(\phi, \Sigma)$ is non-empty if and only if $\phi$ is defined over the residue field $\mathbb{F}_{q}(v)$ and $\Sigma_{v}$ is contained in the image $\phi\left(E_{\phi, v}\right)$ (in the special fiber $E_{v}$ ); that is, $\operatorname{Fr}_{v} \subset \mathcal{C}_{\ell}$ if and only if $v \in \mathcal{B}_{n}(\ell)$. 
For every $f=\ell_{1} \cdots \ell_{m} \in \mathcal{F}$, we write $G_{f}$ for the Galois group of the compositum $K_{\ell_{1}} \cdots K_{\ell_{m}} / K$. For every $i$, there is a natural map $G_{f} \rightarrow G_{\ell_{i}}$, and we define $\mathcal{C}_{n} \subset G_{f}$ to be the maximal subset whose image lies in $\mathcal{C}_{\ell_{i}}$ for all $i$. Then $\operatorname{Fr}_{v} \subset \mathcal{C}_{f}$ if and only if $v \in \mathcal{B}_{n}(f)$.

Given $x>0$, let $\mathcal{L}(x)$ denote the $\ell \in \mathcal{L}$ such that $\ell \leq x$, and $\mathcal{F}(x)$ the $f \in \mathcal{F}$ such that $\ell \in \mathcal{L}(x)$ for every $\ell$ which divides $f$. Given $y>x>0$, let $\mathcal{L}(x, y)$ denote the complement $\mathcal{L}(y)-\mathcal{L}(x)$. We write $\mathcal{G}_{n}(x)$ for the $v \in V_{n}$ which are good with respect to every $\ell \in \mathcal{L}(x)$. One way to compute the density of $\mathcal{G}_{n}(x) \subset V_{n}$ is to apply a standard inclusion-exclusion argument and show that

$$
\left|\mathcal{G}_{n}(x)\right|=\sum_{f \in \mathcal{F}(x)} \mu(f)\left|\mathcal{B}_{n}(f)\right|
$$

where $\mu: \mathcal{F} \rightarrow\{ \pm 1\}$ is the Mobius function. Let $\mathcal{B}_{n}(x, y)$ denote the set of $v \in V_{n}$ which lie in $\mathcal{B}_{n}(\ell)$ for some $\ell \in \mathcal{L}(x, y)$. We make the trivial observation that $\mathcal{B}_{n}(\ell), \mathcal{B}_{n}(\ell, \infty)$ are empty for $\ell>q^{n}+2 q^{n / 2}+1$, because $\ell$ must divide the order of $E_{v}$, hence $\left|\mathcal{G}_{n}\right|=\left|\mathcal{G}_{n}(x)\right|$ for $x$ sufficiently large. On the other hand, for any $x>0$, one can still show that

$$
\left|\mathcal{G}_{n}\right| \geq\left|\mathcal{G}_{n}(x)\right|-\left|\mathcal{B}_{n}(x, \infty)\right|
$$

In general, the expected density of $\mathcal{B}_{n}(f) \subset V_{n}$ is given by the constant $\delta_{n}(f)=$ $\left|\mathcal{C}_{f}\left(q^{n}\right)\right| /\left|G_{f}\left(q^{n}\right)\right|($ cf. theorem 6$)$, so we write

$$
\left|\mathcal{B}_{n}(f)\right|=\delta_{n}(f)\left|V_{n}\right|+\varepsilon_{f} .
$$

Similarly, the expected density of $\mathcal{G}_{n}(x) \subset V_{n}$ is given by $\Delta_{n}(x)=\prod_{\ell \in \mathcal{L}(x)}(1-$ $\left.\delta_{n}(\ell)\right)$, so we write

$$
\left|\mathcal{G}_{n}(x)\right|=\Delta_{n}(x)\left|V_{n}\right|+\varepsilon(x) .
$$

A priori $\mathcal{G}_{n}(x)$ and $\Delta_{n}(x)$ depend on $\mathcal{L}$, but $\mathcal{L}$ is fixed for the entire section, so we omit the dependence from the notation. By the results of 2.3 it follows that $\delta_{n}(\ell)$ is bounded above and below by multiples of $1 /\left(\ell^{r+1} m_{n}(\ell)\right)$, where $m_{n}(\ell)$ is the order of the multiplicative group generated by $q^{n} \bmod \ell$, which was denoted $\operatorname{det}(\Gamma)$ in 2.3. In fact, one can explicitly write down $\delta_{n}(\ell)$ in terms of these quantities. It follows from this estimate that $\Delta(x)$ converges as $x \rightarrow \infty$ and we define $\delta_{n}(\Sigma, \mathcal{S})$, appearing in theorem 1 , as the limit. We further define $a, b$ as the lower and upper bound for this limit obtained from the bound just mentioned for $\delta_{n}(\ell)$ and the estimates $1 \leq m_{n}(\ell) \leq l-1$.

We estimate $|\varepsilon(x)|$ using the identity $\varepsilon(x)=\sum_{f \in \mathcal{F}(x)} \mu(f) \varepsilon_{f}$, and in turn, we estimate $\left|\varepsilon_{f}\right|$ using the following lemma. 
Lemma 6. For every $f \in \mathcal{F}$, we have

$$
\varepsilon_{f}=\mathcal{B}_{n}(f)-\delta_{n}(f)\left|V_{n}\right|=o\left(f^{(r+2) / 2} q^{n / 2} / n\right) .
$$

Proof. The lemma is an application of theorem 6 to $K_{f} / K$. Applying the result of sections 2.3 and 2.4 , we see that $\left|\mathcal{C}_{f}\left(q^{n}\right)\right| \leq 2 f^{r+2}$, for every $f \in \mathcal{F}(x)$.

The lemma is useful only when $x$ is sufficiently small. In fact, it suffices to take $x=x_{n}=n \log (q) /(2 r+6)$.

Corollary 2. $\left|\varepsilon\left(x_{n}\right)\right|=o\left(q^{n} / n\right)$.

Proof. For every $f \in \mathcal{F}\left(x_{n}\right)$, we note that

$$
\log (f) \leq \sum_{\ell \in \mathcal{L}\left(x_{n}\right)} \log (\ell) \leq\left|\mathcal{L}\left(x_{n}\right)\right| \log \left(x_{n}\right) \leq x_{n}+o\left(x_{n}\right),
$$

and in particular, $\log (f) \leq 2 x_{n}$ for $n$ sufficiently large. Applying this to the error term of the lemma gives

$$
\left|\varepsilon_{f}\right|=o\left(f^{(r+2) / 2} q^{n / 2} / n\right)=o\left(q^{n(r+2) /(2 r+6)} q^{n / 2} / n\right)=o\left(q^{n(2 r+5) /(2 r+6)} / n\right) .
$$

We also note that $\left|\mathcal{F}\left(x_{n}\right)\right|=2^{\left|\mathcal{L}\left(x_{n}\right)\right|} \leq e^{x_{n}}=q^{n /(2 r+6)}$, hence

$$
\left|\varepsilon\left(x_{n}\right)\right| \leq \sum_{f \in \mathcal{F}\left(x_{n}\right)}\left|\varepsilon_{f}\right|=q^{n /(2 r+6)} \cdot o\left(q^{n(2 r+5) /(2 r+6)} / n\right)=o\left(q^{n} / n\right) .
$$

By the corollary we have $\Delta_{n}(x)\left|V_{n}\right|-\delta_{n}(\Sigma, \mathcal{S})\left|V_{n}\right|=o\left(q^{n} / n\right)$ and thus, to complete the proof of theorem 1 , it suffices to show that $\mathcal{B}_{n}\left(x_{n}, \infty\right)=o\left(q^{n} / n\right)$, because then

$$
\left|\mathcal{G}_{n}\right|=\left|\mathcal{G}_{n}\left(x_{n}\right)\right|+o\left(q^{n} / n\right)=\delta_{n}(\Sigma, \mathcal{S})\left|V_{n}\right|+o\left(q^{n} / n\right) .
$$

We proceed in three stages by defining $y_{n}=q^{n / 4} / \log \left(q^{n}\right)$ and $z_{n}=q^{n / 4} \log \log \left(q^{n}\right)$, decomposing $\mathcal{L}\left(x_{n}, \infty\right)$ into three disjoint intervals

$$
\mathcal{L}\left(x_{n}, \infty\right)=\mathcal{L}\left(x_{n}, y_{n}\right) \cup \mathcal{L}\left(y_{n}, z_{n}\right) \cup \mathcal{L}\left(z_{n}, \infty\right),
$$

and utilizing the inequality

$$
\left|\mathcal{B}_{n}\left(x_{n}, \infty\right)\right| \leq\left|\mathcal{B}_{n}\left(x_{n}, y_{n}\right)\right|+\left|\mathcal{B}_{n}\left(y_{n}, z_{n}\right)\right|+\left|\mathcal{B}_{n}\left(z_{n}, \infty\right)\right| .
$$

We complete the proof by showing, in the following three lemmas, that each of the terms on the right are $o\left(q^{n} / n\right)$. In each case we use the inequality

$$
\left|\mathcal{B}_{n}(x, y)\right| \leq \sum_{\ell \in \mathcal{L}(x, y)}\left|\mathcal{B}_{n}(\ell)\right|
$$


but we must use different arguments to bound the sum on the right.

Lemma 7 (Small $\ell$ ). $\left|\mathcal{B}_{n}\left(x_{n}, y_{n}\right)\right|=o\left(q^{n} / n\right)$.

Proof. For every $\ell \in \mathcal{L}\left(x_{n}, y_{n}\right)$, we fix a cyclic $\ell$-isogeny $\phi: E_{\phi} \rightarrow E$. We note that, by theorem 2 of $[\mathrm{CH}]$, there is a constant $\ell_{0}=O(1)$ such that $\Gamma=$ $\operatorname{Gal}(K(E[\ell]) / K)$ contains $\mathrm{SL}_{2}(\mathbb{Z} / \ell)$ for every $\ell \neq p, \ell>\ell_{0}$. We may assume, without loss of generality, that $x_{n} \geq \max \left\{\ell_{0}, p\right\}$. The implied constant may be chosen, depending only on $\operatorname{genus}(K)$ and $\operatorname{deg}(S)$, to account for the failure of this assumption, and there are only finitely many $n$ and degenerate $\Gamma$ one must worry about. We write $B \subset \Gamma$ for the Borel subgroup corresponding to $\phi$ and identify it with $\operatorname{Gal}(K(E[\ell]) / K(\phi))$. For every $w \in|K(\phi)|$, we write $\operatorname{Fr}_{w} \subset B$ for the Frobenius conjugacy class.

If $\ell$ does not divide $q^{n}-1$, then we showed in section 2.3 that, for every $v \in \mathcal{B}_{n}(\ell)$, there is a unique $w \in|K(\phi)|$ of degree $n$ and lying over $v$ such that $\operatorname{Fr}_{w} \subset \mathcal{C}(\phi) \subset B$. For $i=1, \ldots, r$, we write $\mathcal{B}_{n, i}(\ell)$ for the subset of $v \in \mathcal{B}_{n}(\ell)$ such that $w$ splits completely in $K\left(\phi, P_{i} / \phi\right)$. In particular, applying theorem 5 we have

$$
\left|\mathcal{B}_{n}(\ell)\right| \leq \sum_{i=1}^{r}\left|\mathcal{B}_{n, i}(\ell)\right|=r\left(q^{n} / \ell^{2}+O\left(\ell q^{n / 2}\right)\right) / n .
$$

On the other hand, if $\ell$ divides $q^{n}-1$ and $v \in \mathcal{B}_{n}(\ell)$, then $\operatorname{Fr}_{v}=\{1\} \subset \Gamma$. Therefore $v$ splits completely in $K\left(\phi, E_{\phi}[\phi]\right) / K$, and theorem 5 implies

$$
\left|\mathcal{B}_{n}(\ell)\right| \leq r\left(q^{n} /\left(\ell^{2}+\ell\right)+O\left(\ell q^{n / 2}\right)\right) / n \leq r\left(q^{n} / \ell^{2}+O\left(\ell q^{n / 2}\right)\right) / n .
$$

Combining the results for all $\ell \in \mathcal{L}\left(x_{n}, y_{n}\right)$ we have

$$
\left|\mathcal{B}_{n}\left(x_{n}, y_{n}\right)\right| \leq \sum_{\ell \in \mathcal{L}\left(x_{n}, y_{n}\right)}\left|\mathcal{B}_{n}(\ell)\right| \leq r\left(q^{n}\left(\sum_{\ell \in \mathcal{L}\left(x_{n}, y_{n}\right)} 1 / \ell^{2}\right)+\left(y_{n}-x_{n}\right) O\left(y_{n} q^{n / 2}\right)\right) / n .
$$

We note that $\sum_{\ell \in \mathcal{L}\left(x_{n}, y_{n}\right)} 1 / \ell^{2}=o(1)$, because $x_{n}$ tends to infinity as $n$ does, and $y_{n}=o\left(q^{n / 4}\right)$, hence $\left|\mathcal{B}_{n}\left(x_{n}, y_{n}\right)\right|=o\left(q^{n} / n\right)$ as desired.

Lemma 8 (Medium $\ell)$. $\left|\mathcal{B}_{n}\left(y_{n}, z_{n}\right)\right|=o\left(q^{n} / n\right)$.

Proof. We fix $\ell \in \mathcal{L}\left(y_{n}, z_{n}\right)$ and use the notation of the previous lemma. If $\ell$ does not divide $q^{n}-1$ and $v \in \mathcal{B}_{n}(\ell)$, then we let $w \in|K(\phi)|$ be the canonical point over $v$ as before. We write $\mathcal{B}_{n, i}^{\prime}(\ell)$ for the subset of $v \in \mathcal{B}_{n}(\ell)$ such that $w$ splits completely in $K\left(\phi, E_{\phi}[\phi]\right)$. Applying theorem 5 gives

$$
\left|\mathcal{B}_{n, i}^{\prime}(\ell)\right|=\left(q^{n} / \ell+O\left(\ell q^{n / 2}\right)\right) / n .
$$


On the other hand, if $\ell$ divides $q^{n}-1$, we let $\mathcal{B}_{n, i}^{\prime}(\ell)$ be the subset of $v \in V_{n}$ which split completely in $K\left(\phi, E_{\phi}[\phi]\right)$. By another application of theorem 5 we have

$$
\left|\mathcal{B}_{n, i}^{\prime}(\ell)\right|=\left(q^{n} / \ell^{2}+O\left(q^{n / 2}\right)\right) / n \leq\left(q^{n} / \ell+O\left(\ell q^{n / 2}\right)\right) / n .
$$

Combining the results for all $\ell \in \mathcal{L}\left(y_{n}, z_{n}\right)$ gives

$$
\left|\mathcal{B}_{n}\left(y_{n}, z_{n}\right)\right| \leq\left(q^{n}\left(\sum_{l \in \mathcal{L}\left(y_{n}, z_{n}\right)} 1 / \ell\right)+\left|\mathcal{L}\left(y_{n}, z_{n}\right)\right| O\left(\ell q^{n / 2}\right)\right) / n .
$$

Using the standard estimate $\sum_{\ell \leq x} 1 / \ell=\log \log (x)+c+o(1)$, where $c$ is a constant, gives

$$
\begin{aligned}
\sum_{l \in \mathcal{L}\left(y_{n}, z_{n}\right)} 1 / \ell & =\log \log \left(z_{n}\right)-\log \log \left(y_{n}\right)+o(1) \\
& =\log \left(\frac{n \log (q)+4 \log \log \log \left(q^{n}\right)}{n \log (q)-4 \log \log \left(q^{n}\right)}\right)+o(1)=o(1) .
\end{aligned}
$$

By the prime number theorem,

$$
\left|\mathcal{L}\left(y_{n}, z_{n}\right)\right| \leq\left|\mathcal{L}\left(z_{n}\right)\right| \leq z_{n} / \log \left(z_{n}\right)+o\left(z_{n} / \log \left(z_{n}\right)\right),
$$

hence

$$
\left|\mathcal{L}\left(y_{n}, z_{n}\right)\right| O\left(\ell q^{n / 2}\right)=O\left(\frac{4\left(\log \log \left(q^{n}\right)\right)^{2}}{\log \left(q^{n}\right)+4 \log \log \log \left(q^{n}\right)} q^{n}\right)=o\left(q^{n}\right) .
$$

This entails that $\left|\mathcal{B}_{n}\left(y_{n}, z_{n}\right)\right|=o\left(q^{n} / n\right)$, as desired.

Lemma 9 (Large $\ell) \cdot\left|\mathcal{B}_{n}\left(z_{n}, \infty\right)\right|=o\left(q^{n} / n\right)$, for $r \geq 6$.

Proof. For every $v \in V$, let $\Sigma_{v}$ denote the image of $\Sigma$ in $E_{v}$. Just as in the number field case, $\Sigma$ is endowed with a quadratic form given by the canonical height pairing and we can argue in the same way as lemma 14 of [GM] to obtain that the number of $v \in V$ such that $\left|\Sigma_{v}\right|<y$ is $O\left(y^{(r+2) / r}\right)$, where the implied constant depends on the regulator $R=\operatorname{det}(\Sigma)$. Their proof actually proves more, namely that the sum of $\operatorname{deg} v$ over the $v$ 's with $\left|\Sigma_{v}\right|<y$ is $O\left(y^{(r+2) / r}\right)$. From the definition it follows that, for every $v \in \mathcal{B}_{n}\left(z_{n}, \infty\right)$, we have

$$
\left|\Sigma_{v}\right|=O\left(q^{n} / z_{n}\right)=o\left(q^{3 n / 4}\right),
$$

therefore

$$
\left|\mathcal{B}_{n}\left(z_{n}, \infty\right)\right|=o\left(\left(q^{3 n / 4}\right)^{1+2 / r}\right) / n=o\left(q^{(3 r+6) n / 4 r} / n\right) .
$$

The lemma follows by observing that $(3 r+6) / 4 r \leq 1$ if $r \geq 6$. 


\section{REFERENCES}

[CH] A. Cojocaru and C. Hall, "Uniform Results for Serre's Theorem for Elliptic Curves," Int. Math. Res. Not. 2005 (2005), no. 50, 3065-3071.

[GM] R. Gupta and M. Ram Murty, "Primitive points on elliptic curves," Compositio Math. 58 (1986), no. 1, 13-44.

[KM] N.M. Katz and B. Mazur, Arithmetic moduli of elliptic curves, Annals of Mathematics Studies, 108. Princeton University Press, Princeton, NJ, 1985.

[LT] S. Lang and H. Trotter, "Primitive points on elliptic curves," Bull. Am. Math. Soc. 83 (1977), 289-292.

[MS] V.K. Murty, J. Scherk, "Effective versions of the Chebotarev density theorem for function fields," C.R. Acad. Sci. Paris, t. 319. Série I, 1994, pp. 523-528.

[U] D.L. Ulmer, "Elliptic curves with large rank over function fields," Annals of Math. 155 (2002), 295-315.

[V] J.F. Voloch, "Primitive points on constant elliptic curves over function fields," Bol. Soc. Brasil. Mat. 21 (1990), 91-94.

Chris Hall

Department of Mathematics

University of Texas, Austin, TX 78712, USA

E-mail: cjh@math.utexas.edu

José Felipe Voloch

Department of Mathematics

University of Texas, Austin, TX 78712, USA

E-mail: voloch@math.utexas.edu 